\title{
OPEN WINDOW THORACOSTOMY FOLLOWED BY INTRATHORACIC FLAP TRANSPOSITION IN THE TREATMENT OF EMPYEMA COMPLICATING PULMONARY RESECTION
}

Jean-François Regnard, MD

Marco Alifano, MD

Philippe Puyo, MD

Estephan Fares, MD

Pierre Magdeleinat, MD

Philippe Levasseur, MD
Objective: Successful treatment of postoperative empyema remains a challenge for thoracic surgeons. We report herein our 12-year experience in the management of this condition by means of open window thoracostomy.

Methods: Open window thoracostomy was used in the treatment of 46 patients with empyema complicating pulmonary resection. A bronchopleural fistula was associated in 39 of 46 cases. Previous operations included pneumonectomy $(\mathrm{n}=30)$, bilobectomy $(\mathrm{n}=5)$, lobectomy $(\mathrm{n}=9)$, and wedge resection $(n=2)$ performed for benign $(n=10)$ or malignant $(n=36)$ disease. In 10 patients open window thoracostomy was definitive because of patient death $(n=2)$, concomitant major illness $(n=2)$, tumor recurrence $(n$ $=4)$, spontaneous closure $(n=1)$, or patient choice $(n=1)$. In 36 cases intrathoracic flap transposition was eventually performed. Muscular ( $\mathrm{n}=$ $29)$, omental $(n=5)$, or combined muscular and omental $(n=2)$ flaps were used to obliterate the thoracostomy cavity and to close a possibly associated bronchopleural fistula. In 9 patients with postpneumonectomy cavities too wide to be filled by the available flaps, a limited thoracoplasty represented an intermediate step.

Results: Among patients treated with definitive open window thoracostomy, local control of the infection was achieved in all the survivors (8/8). After open window thoracostomy and subsequent flap transposition, success (definitive closure of the thoracostomy and, if present, of the bronchopleural fistula) was achieved in $27(75.0 \%)$ of 36 patients. Four initial failures could be salvaged by means of reoperation (initial reopening of thoracostomy and subsequent muscular or omental transposition).

Conclusion: Open window thoracostomy followed by intrathoracic muscle or omental transposition represents a valid therapeutic option in patients with empyema complicating pulmonary resections. (J Thorac Cardiovasc Surg 2000;120:270-5)
$\mathrm{M}$ anagement of empyema complicating pulmonary resections still represents a challenge in thoracic surgery. Empyema is not uncommon as a complication

From Service de Chirurgie Thoracique et Vasculaire, Hôpital Marie Lannelongue, Le Plessis Robinson, France.

Received for publication Oct 25, 1999; revisions requested Dec 6, 1999; revisions received Jan 21, 2000; accepted for publication Feb 28, 2000.

Address for reprints: J. F. Regnard, MD, Service de Chirurgie Thoracique et Vasculaire, Hopital Marie Lannelongue, 135 Av de la Resistance, 92350 Le Plessis Robinson, France (E-mail: jf.regnard@ccml.com).

Copyright (C) 2000 by The American Association for Thoracic Surgery

0022-5223/2000 $\$ 12.00+0 \quad \mathbf{1 2 / 1 / 1 0 6 8 3 7}$

doi:10.1067/mtc.2000.106837 of pneumonectomy, occurring in $2 \%$ to $16 \%$ of cases..$^{1,2}$ Although more uncommon, it may complicate any kind of pulmonary resection. ${ }^{3}$ It is a life-threatening condition with a mortality of approximately $40 \%$ in postpneumonectomy empyema. ${ }^{1-3}$ Empyema is frequently associated with a bronchopleural fistula (BPF) involving the stump of the main bronchus or of a lobar or a segmental bronchus; air leaks may originate also from more peripheral bronchi, as well as from bronchoalveolar fistulas. ${ }^{3}$ The presence of a BPF obviously increases the morbidity and the mortality of empyema by providing a source of continuous contamination of the pleural space and promoting aspiration of infected pleural fluid in the remaining lung. ${ }^{1,4}$ Therefore, the optimal treatment of empyema complicating pulmonary resections 
should take into account the infectious state, the characteristics of the pleural space, and the presence of a fistula. ${ }^{1,5}$

Adequate drainage is the first step in the treatment of patients with postresection empyema. ${ }^{2,5-8}$ Closed tube drainage is generally used in the acute phase, and open drainage is carried out when clinical conditions are stabilized. $^{2,5-7}$ According to other authors, optimal drainage of early postresection empyema is accomplished by reopening of the entire thoracotomy. ${ }^{8}$ On the other hand, several controversies exist concerning the management of a concurrent BPF, as well as the modalities and the timing of definitive chest closure. , $^{2,5},-9$

In the present article we report our experience concerning the treatment of postresection empyema by using open window thoracostomy (OWT) followed by intrathoracic muscular or omental transposition. Such treatment was reserved for patients with persistent empyema after the initial closed drainage.

\section{Patients and methods}

Patients. In the period from 1987-1998, 109 patients were treated in our department for a postoperative empyema; this empyema was associated to BPF in 97 cases.

During the same period, 3889 pulmonary resections were performed in our department. OWT was performed in 46 patients after an initial closed tube drainage for a persistent empyema.

Previous operations included pneumonectomy $(\mathrm{n}=30)$, bilobectomy ( $\mathrm{n}=5)$, lobectomy $(\mathrm{n}=9)$, and wedge resection $(\mathrm{n}=2)$. Indications for pulmonary resection had been lung cancer $(n=36)$ or benign diseases $(n=10)$. Among patients with lung cancer, the final pathologic stage was I in 6, II in 19 , and IIIA in 11 patients. Delay between resection and empyema ranged from 5 days to 45 years (median, 32 days). A BPF was present in 39 of 46 patients (Table I). Pseudomonas aeruginosa, Staphylococcus aureus, and Proteus mirabilis represented the microorganisms most frequently isolated from empyema fluid specimens.

In all the patients, empyema was diagnosed on the basis of clinical data, radiographic appearance, and laboratory examination of pleural fluid. All the patients underwent thoracic computed tomographic scan. A fiberoptic bronchoscope was used to search for an associated BPF.

Pleural drainage. In all patients, closed chest tube thoracostomy was initially performed to drain the purulent collection and, in patients with BPF, to prevent aspiration by the other lung. In the majority of cases, tube thoracostomy, appropriate antibiotics, and supportive care allowed a gradual improvement of clinical conditions.

In case of failure of closed drainage, OWT was planned to achieve optimal pleural drainage and to allow hospital discharge of patients. The delay between pulmonary resection and OWT ranged from 7 days to 45 years (median, 67 days). The site of OWT was selected on the basis of imaging stud-
Table I. Characteristics of subjects included in the study according to the treatment modality

\begin{tabular}{lccc}
\hline & $\begin{array}{c}\text { Definitive } \\
\text { OWT }\end{array}$ & $\begin{array}{c}\text { OWT plus } \\
\text { flap transposition }\end{array}$ & Overall \\
\hline Lung cancer & 8 & 28 & 36 \\
Benign disease & 2 & 8 & 10 \\
BPF at presentation & 8 & 31 & 39 \\
Previous pneumonectomy & 7 & 23 & 30 \\
Previous bilobectomy & 1 & 4 & 5 \\
Previous lobectomy & 2 & 7 & 9 \\
Previous wedge resection & - & 2 & 2 \\
\hline
\end{tabular}

ies, with the aim of correctly draining all purulent collection. Care was taken to limit the injury to chest wall muscles. Two to four rib segments (generally not more than $10 \mathrm{~cm}$ long) were resected to widely expose the empyema cavity. The skin was advanced and brought down to the parietal pleura. Pus and necrotic debris were gently removed, and the cavity was filled with povidone iodine-soaked gauze. The dressings were initially changed once daily and, after discharge, every 2 days. Mean hospital stay after OWT was 8 days. Elimination of gangrenous material and reduction of the pleural cavity were achieved in all the cases; in 21 of 39 patients, spontaneous closure of BPF occurred (Table II).

In 10 ( 8 men and 2 women; mean age, 63.5 years; age range, $42-77$ years) of 46 patients, OWT was definitive, and the second step of the treatment (myoplasty or omentopexy) was never performed because of patient death, concomitant major illness, locoregional or metastatic tumor recurrence, spontaneous closure, or patient choice. Thirty-six patients (32 men and 4 women; mean age, 61.2 years; age range, 37-79 years) underwent eventual intrathoracic muscular or omental transposition (Table I).

Flap transposition. The OWT was not closed by the second step of the Clagett procedure (ie, obliteration of the pleural cavity with an antibiotic solution and skin closure by direct approximation of edges). ${ }^{10}$ In the presence of a BPF, such an approach is not feasible; furthermore, in the absence of a BPF, the risk of empyema recurrence is high. ${ }^{7}$ We preferred to use intrathoracic flap transposition to close the OWT. It was considered possible if no sign of suppuration existed and abundant granulation tissue was present. The presence of a persistent BPF (as observed in 14/36 cases) did not represent a contraindication for closure. Satisfactory clinical conditions and, for patients previously operated on for lung cancer, no evidence of tumor recurrence at restaging were also mandatory. The timing of closure was also influenced by the patient's preferences. Delay between creation and closure of the OWT ranged from 1 to 36 months (median, 6 months).

Intrathoracic muscular transposition was the procedure of choice for closure of OWT and was carried out in 29 patients. If sufficient muscular bulk was available, an omental flap was used alone $(\mathrm{n}=5)$ or in association with muscular flaps $(\mathrm{n}=2)$. 
Table II. Spontaneous closure of BPFs after OWT

\begin{tabular}{lcc}
\hline & $\begin{array}{c}\text { No. of } \\
\text { initial BPFs }\end{array}$ & $\begin{array}{c}\text { Spontaneous closure } \\
\text { of BPFs }\end{array}$ \\
\hline Pneumonectomy $(\mathrm{n}=30)$ & 24 & 16 \\
Bilobectomy $(\mathrm{n}=5)$ & 4 & 2 \\
Lobectomy $(\mathrm{n}=9)$ & 9 & 3 \\
Wedge resection $(\mathrm{n}=2)$ & 2 & 0 \\
\hline
\end{tabular}

When a postpneumonectomy pleural space was judged too large to be filled by the available flaps, a limited extraperiosteal thoracoplasty represented an intermediate step. It was necessary in 9 patients and carried out through a posterolateral incision, thus always preserving the pectoralis major muscle, as well as the serratus anterior muscle.

To perform the muscular flap transposition, we used an incision that included in its trajectory the previous thoracostomy; skin flaps adequate for closure were obtained whenever possible. The muscles to be used for transposition were chosen by taking into account anatomic considerations, as well as the entity of damage caused by previous operations. Single or double muscular flaps were prepared (Table III); extreme care was used to avoid injuries to the principal vascular pedicles of the selected muscles. The flaps were passed into the cavity through the thoracostomy. If a bronchial fistula was still present, it was closed by suturing the muscle at its edges. In most cases the muscular flap was sufficient to obliterate the cavity entirely.

To perform the omentopexy, we created a large omental pedicle by dividing the left or right gastroepiploic vessels and detaching the gastroepiploic arch from the greater curvature of the stomach. A pedicle based on the right or left gastroepiploic vessels was used for transposition in the homolateral hemithorax. The omentum was transposed to the thoracic cavity through a substernal tunnel or an opening in the diaphragm. Care was used to avoid compression of omental vessels, as well as to prevent intestinal herniation. In the presence of a BPF, the omentum was sutured to its edges. In some instances omental and muscular flaps were used together to completely obliterate the pleural space (Table III).

\section{Results}

Outcome after OWT alone. OWT was definitive and flap transposition never performed in 10 of 46 patients. The transposition was not carried out because of patient death (one myocardial infarction and one pulmonary embolism, occurring at 1 and 3 months, respectively), concomitant major illness $(\mathrm{n}=2)$, locoregional $(n=2)$ or metastatic $(n=2)$ tumor recurrence, or patient choice $(\mathrm{n}=1)$. In 1 case spontaneous closure occurred. With the exception of the patient who had a spontaneous closure, the survivors were managed indefinitely on an outpatient clinic basis by local care.
Table III. Intrathoracic flaps used

\begin{tabular}{lr}
\hline Single flap & \\
$\quad$ Pectoralis major & 15 \\
Serratus anterior & 1 \\
Latissimus dorsi & 6 \\
Omentum & 5 \\
Double flap & \\
$\quad$ Pectoralis major plus serratus anterior & 4 \\
Latissimus dorsi plus serratus anterior & 2 \\
Latissimus dorsi plus rhomboid & 1 \\
Latissimus dorsi plus omentum & 1 \\
Pectoralis major plus omentum & 1
\end{tabular}

Local control of the infection was satisfactory in all the patients, and septicemia occurred in no one. Spontaneous closure of BPF occurred in 5 patients.

Outcome after OWT and subsequent flap transposition. Thirty-three of 36 patients were alive 6 months after the completion of the treatment and available for assessment of treatment success. The causes of the 3 deaths were recurrent empyema with septicemia (treatment failure) in 2 patients and cancer progression in 1 patient. All the deaths occurred more than 1 month after the completion of the treatment and discharge from the hospital. Neither flap necrosis nor other major complications were observed. One wound infection occurred, which was successfully managed by drainage and antibiotics.

In $27(75.0 \%)$ of 36 patients, the intrathoracic flap transposition was successful; no infection recurred, and definitive closure of thoracostomy was achieved. Independent of the size of the residual pleural space at the time of flap transposition, in $9(25.0 \%)$ cases empyema recurred, and reopening of the OWT was necessary. Four of these patients were eventually successfully treated by further intrathoracic muscular (1 patient) or omental (2 patients) transposition; in another patient a thoracoplasty followed by omentopexy was used with success. In the remaining 5 patients no further attempt for closure was possible because of persistent infection and the OWT was definitive. The overall success rate was $86 \%$ (31/36; Table IV).

Among patients with BPF at the time of flap transposition $(\mathrm{n}=14)$, the outcome was immediately favorable in $10(71.4 \%)$; among the 4 whose initial treatment failed, 2 were successfully treated by further operations (Table V).

Among patients without BPF, when flap transposition was performed $(n=22)$, a successful outcome was observed in 17 (77.3\%) patients; two of the patients whose flap transposition was unsuccessful underwent a successful reoperation (Table V). 
Table IV. Results of treatment procedures in patients treated by OWT followed by flap transposition, according to the kind of initial operation

\begin{tabular}{|c|c|c|c|c|c|}
\hline & Initial success & Initial failure & New operation & Success of new operation & Overall success \\
\hline Pneumonectomy $(\mathrm{n}=23)$ & $17(73.9 \%)$ & $6(26.1 \%)$ & 3 & 3 & $20 \quad(86.9 \%)$ \\
\hline Bilobectomy $(n=4)$ & $2 \quad(50 \%)$ & $2 \quad(50 \%)$ & - & - & $(50 \%)$ \\
\hline Lobectomy $(\mathrm{n}=7)$ & $6(85.7 \%)$ & $1(14.3 \%)$ & 1 & 1 & $7 \quad(100 \%)$ \\
\hline Wedge resection $(\mathrm{n}=2)$ & $2(100 \%)$ & $(0 \%)$ & - & - & $2 \quad(100 \%)$ \\
\hline Overall $(\mathrm{n}=36)$ & $27(75.0 \%)$ & $9(25.0 \%)$ & 4 & 4 & $31 \quad(86.1 \%)$ \\
\hline
\end{tabular}

\section{Discussion}

Adequate pleural drainage remains the cornerstone of the initial treatment of empyema complicating pulmonary resections. Tube thoracostomy allows empyema drainage and prevents aspiration in the controlateral lung if a BPF is present. ${ }^{2,5,7}$ In our series, as well as in the experience of several authors, ${ }^{2,5-7}$ closed chest tube drainage represented the initial part of management of postresection empyema and allowed for stabilization of the patients' clinical conditions. However, closed drainage often fails to completely drain the cavity with subsequent unsatisfactory control of the infection; in these cases open drainage is performed. ${ }^{2,5-7}$ Furthermore, open drainage allows for management of patients on an outpatient clinic basis much more easily than closed drainage. In our series, mean hospital stay after OWT was 8 days.

In our study, OWT was carried out according to the standard technique ${ }^{5}$; the entity of rib resection was as limited as possible to ensure adequate drainage and easy changes of dressings. In these severely ill patients we preferred to limit the surgical trauma as much as possible, and therefore no attempt was made to close a $\mathrm{BPF}$ when present. This approach is probably responsible for the low incidence of complications observed in the present series. Furthermore, similar to others' experience, ${ }^{5}$ in $53.8 \%$ of cases, the BPF closed spontaneously after OWT, probably because of improvement (or resolution) of the infective condition of the pleural cavity. Other authors have advocated a more aggressive management of early postresection empyema and BPF, as well as different modalities of OWT closure. ${ }^{6,8,11}$ Pairolero and colleagues ${ }^{8}$ suggested immediate open pleural drainage by reopening of the entire thoracotomy incision. In the presence of BPF they performed restapling of long bronchial remnants (if present) or complete reopening and resuture of short stumps. Immediate muscular transposition is used to protect the suture line or to cover the fistula if resuture is not possible. ${ }^{8}$ They suggested OWT closure with the second stage of the Clagett procedure. ${ }^{10}$ By using this approach, Pairolero and colleagues ${ }^{8}$ reported a successful outcome in $26(57.8 \%)$ of 45 patients; 6 $(13.3 \%)$ operative deaths were also observed, and a mean of 5 surgical procedures were necessary. It is noteworthy that the second stage of the Clagett procedure was followed by empyema recurrence in 5 $(16.1 \%)$ of 31 evaluable patients. ${ }^{8}$ On the other hand, several authors have used the 2-step Clagett procedure (without flap transposition) in the treatment of postresection empyema. This technique is possible only in the absence of BPF, and the results are very variable in different authors' experience. In the series of Stafford and Clagett, ${ }^{12}$ sterilization of empyema and definitive closure of the OWT were accomplished in 11 (61\%) of 18 patients at the first attempt. Goldstraw ${ }^{2}$ reported successful results in $17(77.3 \%)$ of 22 patients in whom the entire procedure could be completed, whereas in the experience of Shamji and colleagues, ${ }^{7}$ in only 2 of 5 patients who underwent the second step of the procedure was a permanent closure of the OWT achieved.

Gharagozloo and colleagues ${ }^{13}$ have recently reported a surprisingly high success rate $(100 \%)$ in patients with early postpneumonectomy empyema associated with BPF. They suggested a procedure based on emergency tube drainage followed by thoracotomy, debridement of necrotic tissue, bronchial stump resuture, and immediate closure of the thoracotomy. Postoperative pleural irrigation and obliteration of the space by antibiotic solution constitute the final part of the treatment. The authors speculated that in most patients in their population a technical error in handling the bronchus at the time of initial thoracotomy was probably responsible for the occurrence of the BPF, and therefore they considered a technically satisfactory reclosure of the bronchus of paramount importance in the management of such patients. On the basis of these considerations, we think that presently the approach suggested by Gharagozloo and colleagues should be considered only in a very selected subset of patients.

In our experience after OWT, intrathoracic muscular or omental transposition was used to obliterate the 
Table V. Results of treatment procedures in patients treated by OWT followed by flap transposition, according to the presence or the absence of a BPF on the moment of flap transposition

\begin{tabular}{lccccc}
\hline & Initial success & Initial failure & New operation & Success of new operation & Overall success \\
\hline BPF present $(\mathrm{n}=14)$ & $10(71.4 \%)$ & $4(28.6 \%)$ & 2 & 2 & $12(85.7 \%)$ \\
BPF absent $(\mathrm{n}=22)$ & $17(77.3 \%)$ & $5(22.8 \%)$ & 2 & 2 & $19(86.4 \%)$ \\
Overall $(\mathrm{n}=36)$ & $27(75.0 \%)$ & $9(25.0 \%)$ & 4 & 4 & $31(86.1 \%)$ \\
\hline
\end{tabular}

pleural space and, at the same time, to close a possibly associated BPF. It has been reported that both omentum $^{14,15}$ and muscle $e^{5,16,17}$ flaps may play an important role in infection control. Furthermore, both stimulate neoangiogenesis of ischemic bronchial stumps. ${ }^{18-20}$ In the present study a satisfactory success rate was also observed among patients with persistent BPF at the time of flap transposition.

The choice of muscles for transposition depends on the location of the space to be filled, as well as on previous operations. Pectoralis major is the muscle of choice for filling anterior cavities because of its bulk and integrity after a posterolateral thoracotomy. It is a general policy in our institution to respect the serratus anterior in all thoracotomies, and therefore this muscle is almost always available for transposition. The latissimus dorsi has also been used in several instances in our series. If it had been preserved at the time of the initial operation - if an anterior thoracotomy or a musclesparing lateral thoracotomy had been carried out-it can be mobilized and transposed entirely. On the other hand, it is generally believed that a previously divided latissimus dorsi will survive in situ, but the entire muscle will not survive as a proximally based muscle flap. ${ }^{21}$ Furthermore, when a single muscle is not sufficient, 2 or more muscles may be used, as in 8 patients in our series.

In 9 patients in our series a limited extraperiosteal thoracoplasty represented an intermediate step between OWT and flap transposition. We did not carry out the thoracoplasty and flap transposition at the same operative time to limit the surgical trauma in these weak patients. In our opinion, thoracoplasty should be discouraged as the definitive and only procedure for treatment of postoperative empyema. When used alone, extensive rib resection (full thoracoplasty) is generally required, with a subsequent high operative mortality $^{22,23}$ and significant physiologic changes negatively affecting the function of the controlateral lung. ${ }^{23}$ Furthermore, the success rates reported are not completely satisfactory. Among 16 patients with BPF complicating resection for lung cancer, Peppas and colleagues $^{22}$ observed 4 hospital deaths and 2 treatment failures. Young and Ungerleider ${ }^{24}$ reported an operative mortality of $10 \%$ and $18 \%$ of survivors with persistent empyema, BPF, or both.

Our results are in agreement with those reported in previous studies in which similar techniques and timing were used in smaller series of patients. ${ }^{5,9}$ In particular, Cicero and colleagues ${ }^{9}$ reported good results in 2 of 2 patients with postresection empyema treated by OWT followed by intrathoracic transposition of myocutaneous flaps; on the other hand, Garcia-Yuste and colleagues ${ }^{5}$ reported a successful outcome in 21 of 22 patients with tuberculous empyema and in 14 of 18 patients with postresection empyema by using OWT followed by intrathoracic muscular transposition.

We believe that OWT followed by intrathoracic flap transposition is a safe and effective technique in the management of empyema complicating pulmonary resections. Compared with the second step of the Clagett procedure, in the absence of BPF at the time of OWT closure, intrathoracic flap transposition provided results similar to those reported by some authors ${ }^{2,12}$ but remarkably better than those shown by others. ${ }^{7}$ Therefore, no definitive conclusion can be drawn. On the other hand, in the presence of a persistent BPF, the second step of the Clagett procedure is not feasible, and flap transposition represents a very effective tool.

\section{REFERENCES}

1. Kopec SE, Irwin RS, Umali-Torres CB, Balikian JP, Conlan AA. The postpneumonectomy state. Chest 1998;114:1158-84.

2. Goldstraw P. Treatment of postpneumonectomy empyema: the case for fenestration. Thorax 1979;72:319-22.

3. Deschamps C, Allen MS, Trastek VF, Pairolero PC. Empyema following pulmonary resection. Chest Surg Clin North Am 1994;4:583-92.

4. Weber J, Grabner D, Al-Zand K, Beyerd D. Empyema after pneumonectomy. Empyema window or thoracoplasty? Thorac Cardiovasc Surg 1990;38:355-8.

5. Garcia-Yuste M, Ramos G, Duque JL, et al. Open-window thoracostomy and thoracomyoplasty to manage chronic pleural empyema. Ann Thorac Surg 1998;65:818-22.

6. Deschamps C, Pairolero PC, Allen MS, Trastek VF. Management of postpneumonectomy empyema and bronchopleural fistula. Chest Surg Clin North Am 1996;6:519-27.

7. Shamiji FM, Ginsberg RJ, Cooper JD. Open window thoracosto- 
my in the management of postpneumonectomy empyema with or without bronchopleural fistula. J Thorac Cardiovasc Surg 1983;86:818-22.

8. Pairolero PC, Arnold PG, Trastek VF, Meland BN, Kay PP. Postpneumonectomy empyema: the role of intrathoracic muscle transposition. J Thorac Cardiovasc Surg 1990;99:958-68.

9. Cicero R, del Vecchyo C, Porter JK, Carreno J. Open window thoracostomy and plastic surgery with muscle flaps in the treatment of chronic empyema. Chest 1986;89:374-7.

10. Clagett OT, Geraci JE. A procedure for the management of postpneumonectomy empyema. J Thorac Cardiovasc Surg 1963;45:141-5.

11. Kalweit G, Feindt P, Huwer H, Volkkmer I, Gams E. The pectoral muscle flaps in the treatment of bronchial stump fistula following pneumonectomy. Eur J Cardiothorac Surg 1994;8:358-62.

12. Stafford EG, Clagett OT. Postpneumonectomy empyema: neomycin instillation and definitive closure. J Thorac Cardiovasc Surg 1972;63:771-5.

13. Gharagozloo F, Trachiotis G, Wolfe A, DuBree KJ, Cox JL. Pleural space irrigation and modified Clagett procedure for the treatment of early postpneumonectomy empyema. J Thorac Cardiovasc Surg 1998;116:943-8.

14. Puskas JD, Mathisen DJ, Grillo HC, Wain JC, Wright CD, Moncure AC. Treatment strategies for bronchopleural fistula. J Thorac Cardiovasc Surg 1995;109:989-96.

15. Goldsmith H, De Los Santos R, Beattie E. Relief of chronic lymphedema by omental transposition. Ann Surg 1967;166:573-85.
16. Mathes SJ, Alpert BS, Chang N. Use of the muscle flap in chronic osteomyelitis: experimental and clinical correlation. Plast Reconstr Surg 1982;69:815-29.

17. Arnold PG, Pairolero PC. Intrathoracic muscle flaps: an account of their use in the management of 100 consecutive patients. Ann Surg 1990;211:656-60.

18. Morgan E, Lima O, Goldberg M, Ferdman A, Luk S, Cooper J. Successful revascularization of totally ischemic bronchial autograft with omental pedicle flaps in dogs. J Thorac Cardiovasc Surg 1982;84:204-10.

19. Goldsmith H, Griffith A, Kupferman A, Catsimpoolas N. Lipid angiogenic factor from omentum. JAMA 1984;252:2034-6.

20. Fell SC, Mollenkopf FP, Montefusco CM. Revascularization of ischemic bronchial anastomoses by an intercostal pedicle flap. J Thorac Cardiovasc Surg 1985;90:172-8.

21. Harris SU, Nahai F. Intrathoracic muscle transposition: surgical anatomy and techniques of harvest. Chest Surg Clin North Am 1996;6:501-18.

22. Peppas G, Molnar TF, Jeyasingham K, Kirk AB. Thoracoplasty in the context of current surgical practice. Ann Thorac Surg 1993;56:903-9.

23. Barker WL. Thoracoplasty. Chest Surg Clin North Am 1994;4:593-615.

24. Young WG, Ungerleider RM. Surgical approach to the chronic empyema: thoracoplasty. In: Deslauriers J, Lacquet LK, editors. Thoracic surgery: surgical management of pleural disease. St Louis: Mosby; 1990. p. 253. 